\title{
Indicadores sociales y de salud a partir de la implementación de Red Unidos en Bogotá
}

\author{
Social and health indicators since Red Unidos \\ implementation in Bogotá
}

\author{
Gino Montenegro-Martínez ${ }^{1}$; Angela-Maria Segura-Cardona ${ }^{1}$
}

\begin{abstract}
Forma de citar: Montenegro Martínez G, Segura Cardona AM. Indicadores sociales y de salud a partir de la implementación de
\end{abstract} Red Unidos en Bogotá. Salud UIS. 2019; 52(1): 9-19. doi: http://dx.doi.org/10.18273/revsal.v52n1-2020003 @) (1)

\section{Resumen}

Introducción: Red Unidos es la política colombiana para la reducción de la pobreza extrema. Promueve el acceso de los hogares a servicios de salud, educación y a mejorar sus condiciones de habitabilidad, nutrición, ingresos y trabajo. El acceso a servicios, como los promovidos por la Red Unidos puede cambiar en el tiempo el comportamiento de indicadores sociales y de salud. Objetivo: Valorar el comportamiento de indicadores sociales y de salud a partir de la implementación de Red Unidos en Bogotá entre 2002-2017. Materiales y métodos: Se realizó un estudio longitudinal con información retrospectiva. Se tomaron registros de indicadores sociales y de salud para Bogotá durante el periodo 2002 - 2017. Se realizó un análisis de series de tiempo interrumpida para analizar el cambio de tendencia y de nivel de los indicadores sociales y de salud a partir de la implementación de Red Unidos. Resultados: Se presentaron reducciones significativas en el comportamiento de los indicadores de salud por cada año de observación para el periodo de estudio, excepto para la prevalencia de desnutrición crónica. La razón de mortalidad materna y la prevalencia de desnutrición global presentaron cambios significativos de nivel, pero no de tendencia. De manera similar, los indicadores sociales presentaron reducciones significativas por cada año de observación durante el periodo de estudio, a excepción de la tasa global de participación laboral. Los cambios de nivel para este grupo de indicadores no fueron persistentes en el tiempo. Conclusiones: Los cambios en el comportamiento de los indicadores posterior a la implementación de la Red no fueron sostenidos en el tiempo. Sin embargo, estos cambios son producto de múltiples factores, no sólo de la Red Unidos.

Palabras clave: Política pública; Pobreza; Indicadores de salud; Condiciones sociales; Análisis de series de tiempo interrumpido.

\begin{abstract}
Introduction: Red Unidos is the Colombian policy aimed to reduce extreme poverty. Promote the access of households in extreme poverty to health, education and to improve its habitability, nutrition, income and work. Access to services, such as those promoted can change the performance of social and health indicators. Objective: To assess the performance of social and health indicators since Red Unidos implementation in Bogotá 2002-2017. Materials and methods: Longitudinal study with retrospective information was carry out. Health and social indicators from

1. Universidad CES. Medellín, Colombia.

Correspondencia: Gino Montenegro Martínez. Dirección: Calle 10A 22-04 Medellín. Teléfono: (+574) 4440555 ext 1963 . Correo electrónico: gmontenegro@ces.edu.co
\end{abstract}


Bogota between 2002 - 2017 were collected. Interrupted Time Series were used to analyze the change of trend and level of social and health indicators since Red Unidos implementation. Results: Health indicators show significative reductions, but no chronic malnutrition prevalence. Maternal mortality ratio and global prevalence of malnutrition showed significant changes of level, but no trend. Similarly, social indicators showed significant reductions for each year of observation, but no overall participation rate. The level changes of this indicators were not persistent over time. Conclusions: Changes in the post-intervention performance of the indicators were not held. However, its increase or reduction depend on other circumstances that affect their performance but not exclusively result since Red Unidos implementation in Bogotá.

Key words: Public policy; Poverty; Health status indicators; Social conditions; Interrupted time series analysis.

\section{Introducción}

La pobreza es un fenómeno que tiene repercusiones en el ejercicio y protección de los derechos humanos ${ }^{1,2}$. Se relaciona con la exclusión social, la malnutrición y el insuficiente acceso a servicios esenciales como agua, educación, salud y una vivienda digna, entre $\operatorname{otros}^{3-5}$, los cuales repercuten en mala salud ${ }^{6-10}$. Por otro lado, casi 100 millones de personas en el mundo se ven inmersas cada año en condiciones de extrema pobreza a causa de gastos relacionados con la atención en salud ${ }^{11}$. Frente a esta dinámica circular se establece que "la pobreza engendra mala salud y la mala salud mantiene la pobreza"12.

Las causas por las que una familia es pobre se han explicado como resultado de una serie de trampas en donde su incapacidad para acumular capital humano, explicaría la no superación de dicha condición ${ }^{13}$. Por otro lado, Banerjee y Duflo ${ }^{14}$, afirman que la escasa información para tomar decisiones y las precarias condiciones de su entorno próximo no les permite a las personas pobres ser actores activos en el mejoramiento de sus condiciones de vida. Estas dos perspectivas, se entretejen bajo la lógica de la pobreza como cuestión de origen individual, de modo que su solución requiere de esfuerzos individuales.

Por el contrario, para Zibechi ${ }^{15}$ el problema no es la pobreza, es la acumulación de la riqueza que se encuentra alojada y protegida por la estructura misma que la produce y la reproduce. De este modo, la lucha contra la pobreza demanda, entre otros, la configuración de una estructura que permita el reconocimiento y la garantía de los derechos económicos, sociales y culturales $^{16}$. Esta última noción, corresponde a una mirada que no se reduce a la caracterización y al desarrollo de intervenciones desde la situación resultante -la pobreza-, sino que indaga por sus orígenes más orgánicos y a partir de allí plantea alternativas de acción.
La reducción de la pobreza hace parte de la agenda global. El primero de los Objetivos de Desarrollo del Milenio (ODM) se orientó a reducir para el 2015 a la mitad el porcentaje de personas con ingresos menores a $\$ 1,25$ USD $^{17}$. Su renovación, a través de los Objetivos de Desarrollo Sostenible (ODS), establece poner fin a la pobreza extrema y reducir a la mitad las personas que viven en pobreza para $2030^{18}$. Según el Banco Mundial, para el 2015, 736 millones de personas en el mundo vivían con menos de \$1,90 USD diarios. Asia meridional y África subsahariana concentra el 85\% de la población en esta condición. En América Latina y el Caribe afecta al 3,5\%, lo que corresponde a 25,9 millones de personas ${ }^{19}$.

La pobreza afecta en Colombia a una importante proporción de la población. De acuerdo con el Departamento Administrativo Nacional de Estadística (DANE), en términos de pobreza monetaria, en el 2002 el 49,7\% de los colombianos eran pobres, cifra que para el año 2017 se redujo a 26,9\%. Para Bogotá este mismo indicador fue de $31,7 \%$ y $12,4 \%$ para 2010 y 2017 respectivamente. En relación con pobreza extrema monetaria, para el año 2002, el $17,7 \%$ de los colombianos estaba en esta condición y para el 2017 se redujo al 7,4\%. En Bogotá para el 2002 el 7,1\% de la población estaba en pobreza extrema, para el 2017 este indicador llegó al 2,4\% ${ }^{20}$.

En el 2006 Colombia estableció la Red de Protección Social contra la Pobreza Extrema, Red Juntos ${ }^{21}$. Para el 2011 se renovó la estrategia y se denominó Red Unidos $^{22}$. La Red está conformada por diversos actores y sectores del Estado y la sociedad que, desde un enfoque de promoción social y entendiendo la pobreza como un fenómeno multidimensional, promueven el acceso de los hogares en pobreza extrema a servicios de salud y educación y a mejorar sus condiciones de habitabilidad, nutrición y trabajo, entre otros, los cuales se definieron como centrales para la superación de su condición ${ }^{21-23}$. 
Red Unidos inicia su implementación en el año 2007 en Buenaventura con la participación de dos mil mujeres cabeza de familia. Para ese momento, se seleccionaron otros 33 municipios como parte de una prueba piloto. Posteriormente, entre el 2009 y el 2010 se expandió la estrategia a nivel nacional ${ }^{24}$. De acuerdo con el informe de cumplimiento de logros del Departamento de la Prosperidad Social, con corte a 30 de abril de 2017, estaban siendo acompañados 628,751 hogares colombianos.

La implementación en Bogotá se inició en el año 2010, para ese momento los hogares acompañados eran aquellos ubicados en barrios o localidades con mayores niveles de pobreza clasificados a partir del Sistema de Identificación de Potenciales Beneficiarios de Programas Sociales (SISBEN). En el 2015 se implementó el modelo Urbano U-100, donde el acompañamiento se realizó sólo a los hogares beneficiarios de Subsidio Familiar de Vivienda en Especie (SFVE). Según el informe de cumplimiento de logros del departamento de la prosperidad social, al 30 de abril de 2017, se tenían focalizados 4,763 hogares de los cuales se eran acompañados 3,619.

Las políticas se comportan como determinantes de la salud, por lo que suponen un objeto de estudio de especial relevancia para la salud pública. Algunas de ellas están originadas por burocracia no sanitarias, pero contienen acciones que son relevantes para la salud $^{25}$. Este es el caso de Red Unidos, en la medida que promover el acceso a servicios como educación, salud, mejorar la habitabilidad, la nutrición e ingresos y trabajo, presume un cambio de las condiciones de vida.

De este modo, se parte del supuesto de que los hogares que hacen parte de Red Unidos pueden mejorar sus condiciones de vida, ésto a su vez podría reflejarse en un cambio en los indicadores sociales y de salud en Bogotá.

El objetivo de este trabajo es valorar el comportamiento de indicadores sociales y de salud a partir de la implementación de Red Unidos en Bogotá.

\section{Materiales y métodos}

Se realizó un estudio longitudinal con información retrospectiva. Se tomaron registros de indicadores sociales y de salud de Bogotá. para el periodo 2002 - 2017. Su selección se orientó a dar cuenta del comportamiento de dichos indicadores antes y después de la implementación de Red Unidos.
En relación con los indicadores de salud se incluyó la tasa de mortalidad en menores de 5 años, la tasa de mortalidad infantil, la razón de mortalidad materna, la prevalencia de desnutrición global y crónica y la prevalencia de bajo peso al nacer. En relación con los indicadores sociales se tomó la pobreza monetaria, pobreza monetaria extrema, coeficiente de Gini, tasa global de participación laboral, tasa de ocupación y tasa de desempleo.

Se solicitó información a la secretaría distrital de salud de Bogotá, además se buscaron informes oficiales sobre el comportamiento de los resultados de interés. Adicionalmente, se consultaron los módulos de estadísticas vitales, mercado laboral y pobreza y condiciones de vida del DANE ${ }^{16}$ con el fin de contar con la mayor información posible.

La tasa de mortalidad en menores de 5 años se calculó tomando como numerador la cifra de muertes reportadas por el $\mathrm{DANE}^{26}$ y como denominador el total de nacidos vivos para el mismo año. En el caso de la tasa de mortalidad infantil, la razón de mortalidad materna, la prevalencia de desnutrición global y crónica y la prevalencia de bajo peso al nacer, fueron tomados de consultas a la secretaría distrital de salud de Bogotá y del informe sobre metas de los objetivos de desarrollo del milenio 2015 de la secretaria distrital de planeación de Bogotá27.

En relación con los indicadores de pobreza monetaria, pobreza monetaria extrema, coeficiente de Gini, tasa global de participación laboral, tasa de ocupación y de desempleo, fueron tomados del módulo de mercado laboral, pobreza y condiciones de vida del DANE. Para el caso de pobreza monetaria, pobreza monetaria extrema y coeficiente de Gini se imputaron los valores de 2005 y 2006 dado que no se encontró medición para estos años.

Para el análisis estadístico se utilizó la técnica de Análisis de Series de Tiempo Interrumpida (STI). Esta se ha descrito como central para la evaluación de políticas y programas en diferentes ámbitos ${ }^{28}$. Es útil cuando el investigador no controla la implementación ni la asignación de una intervención. Permite valorar los cambios de un resultado de interés antes y después de la implementación ${ }^{29}$. Para ello, analiza los cambios de la variable de interés en comparación con un contrafactual, el cual es representado por la tendencia continua esperada en ausencia de la intervención ${ }^{30}$. 
La valoración del cambio de la variable de interés se realiza a partir de la medición del nivel y/o tendencia. El cambio de nivel hace referencia al punto de intersección para el primer momento $\mathrm{y}$ valor inmediatamente después de la intervención. La tendencia es la tasa de cambio de una medida que puede ser un aumento o una reducción notoria de manera persistente en el tiempo ${ }^{31}$.

Para llevar a cabo el análisis por STI, la autocorrelación, la no estacionariedad y la estacionalidad deben ser consideradas $^{32}$; además, se debe contar con un mínimo de observaciones antes y después de la intervención que permita determinar la magnitud de cambio. Aunque no se tiene un número establecido, este puede variar entre 8 y 12 períodos de datos antes y después de la intervención. Dado que se trata de datos agregados, no se puede inferir los resultados de manera individual ya que se estaría cayendo en la falacia ecológica ${ }^{31}$.

Finalmente, una de las claves en el análisis de STI es predecir cómo se hubiese comportado el resultado de interés si no se hubiera llevado a cabo la intervención. Para ello, se ha descrito la necesidad de contar con una población que funcione como contrafactual. Dado que no es posible observar la intervención implementada y no implementada en la misma población al mismo tiempo y no se puede establecer el mejor contrafactual, no es posible inferir relaciones causales mediante esta técnica de análisis ${ }^{30}$.

En términos de aplicación de la técnica se construyó una matriz para Bogotá con los indicadores respectivos. Las variables tiempo, nivel y tendencia se añadieron a cada uno de los indicadores seleccionados. Posteriormente, se realizaron gráficos de línea con el fin de examinar el comportamiento de cada indicador durante el período de estudio 2002-2017. El período definido como pre-intervención fue 2002-2009 y el período pos-intervención 2010-2017. Seguidamente, se realizó una regresión lineal para cada uno de los indicadores siguiendo el método de mínimos cuadrados ordinarios obteniendo los coeficientes de regresión $(\beta)$.

Se realizó la prueba de Durbin Watson para probar autocorrelación entre los residuos de la regresión inicial. Posteriormente, se graficaron correlogramas simples y parciales para determinar si era necesario realizar una corrección. En tal caso, la corrección se realizó aplicando el método de mínimos cuadrados generalizados. Los análisis y los gráficos se realizaron en el software R Studio (software libre) v. 3.5.2.

\section{Resultados}

La presentación de los resultados se realizó iniciando por los indicadores relacionados con salud y posteriormente de los indicadores sociales. La estimación de los cambios en nivel y tendencia para los indicadores de salud para Bogotá antes y después de la implementación de Red Unidos se encuentran en la Tabla 1. La presentación de las series de tiempo interrumpidas se presenta en el

\section{Gráfico 1.}

Tabla 1. Estimación de cambios de nivel y tendencia de indicadores de salud para Bogotá antes y después de la implementación de Red Unidos.

\begin{tabular}{|c|c|c|c|c|}
\hline Variable & & $\beta$ & IC $95 \%$ & $\mathbf{p}$ \\
\hline \multirow{4}{*}{$\begin{array}{l}\text { Tasa de } \\
\text { mortalidad en } \\
\text { menores de } 5 \\
\text { años por } \\
1000 \mathrm{NV}\end{array}$} & Intercepto & 18,78 & & \\
\hline & Tiempo & $-0,53$ & $-0,76 ;-0,30$ & $<0,001$ \\
\hline & Nivel & $-0,15$ & $-0,16 ; 1,35$ & 0,826 \\
\hline & Tendencia & 0,04 & $-0,27 ; 0,37$ & 0,750 \\
\hline \multirow{4}{*}{$\begin{array}{l}\text { Tasa de } \\
\text { mortalidad } \\
\text { infantil por } \\
1000 \mathrm{NV}\end{array}$} & Intercepto & 16,15 & & \\
\hline & Tiempo & $-0,43$ & $-0,61 ;-0,26$ & $<0,001$ \\
\hline & Nivel & 0,006 & $-1,13 ; 1,14$ & 0,99 \\
\hline & Tendencia & $-0,04$ & $-0,29 ; 0,19$ & 0,68 \\
\hline \multirow{4}{*}{$\begin{array}{l}\text { Razón de } \\
\text { mortalidad } \\
\text { materna por } \\
100,000 \mathrm{NV}\end{array}$} & Intercepto & 70,03 & & \\
\hline & Tiempo & $-3,32$ & $-3,65 ;-2,99$ & 0,00 \\
\hline & Nivel & $-2,61$ & $-4,88 ;-0,35$ & $<0,05$ \\
\hline & Tendencia & 1,51 & 1,$24 ; 1,78$ & 0,00 \\
\hline \multirow{4}{*}{$\begin{array}{l}\text { Prevalencia de } \\
\text { desnutrición } \\
\text { global }\end{array}$} & Intercepto & 8,63 & & \\
\hline & Tiempo & $-0,34$ & $-0,42 ;-0,27$ & $<0,001$ \\
\hline & Nivel & $-0,65$ & $-1,12 ;-0,18$ & $<0,05$ \\
\hline & Tendencia & 0,31 & 0,$21 ; 0,41$ & $<0,001$ \\
\hline \multirow{4}{*}{$\begin{array}{l}\text { Prevalencia de } \\
\text { desnutrición } \\
\text { crónica }\end{array}$} & Intercepto & 22,46 & & \\
\hline & Tiempo & $-0,21$ & $-0,88 ; 0,44$ & 0,49 \\
\hline & Nivel & $-2,10$ & $-3,44 ; 0,55$ & $<0,05$ \\
\hline & Tendencia & 0,50 & $-0,43 ; 1,45$ & 0,72 \\
\hline \multirow{4}{*}{$\begin{array}{l}\text { Prevalencia } \\
\text { de bajo peso } \\
\text { al nacer }\end{array}$} & Intercepto & 11,37 & & \\
\hline & Tiempo & 0,16 & 0,$05 ; 0,27$ & $<0,01$ \\
\hline & Nivel & 0,23 & $-0,48 ; 0,96$ & 0,48 \\
\hline & Tendencia & $-0,21$ & $-0,37 ;-0,59$ & $<0,05$ \\
\hline
\end{tabular}

Fuente: elaboración propia a partir del análisis de los datos 


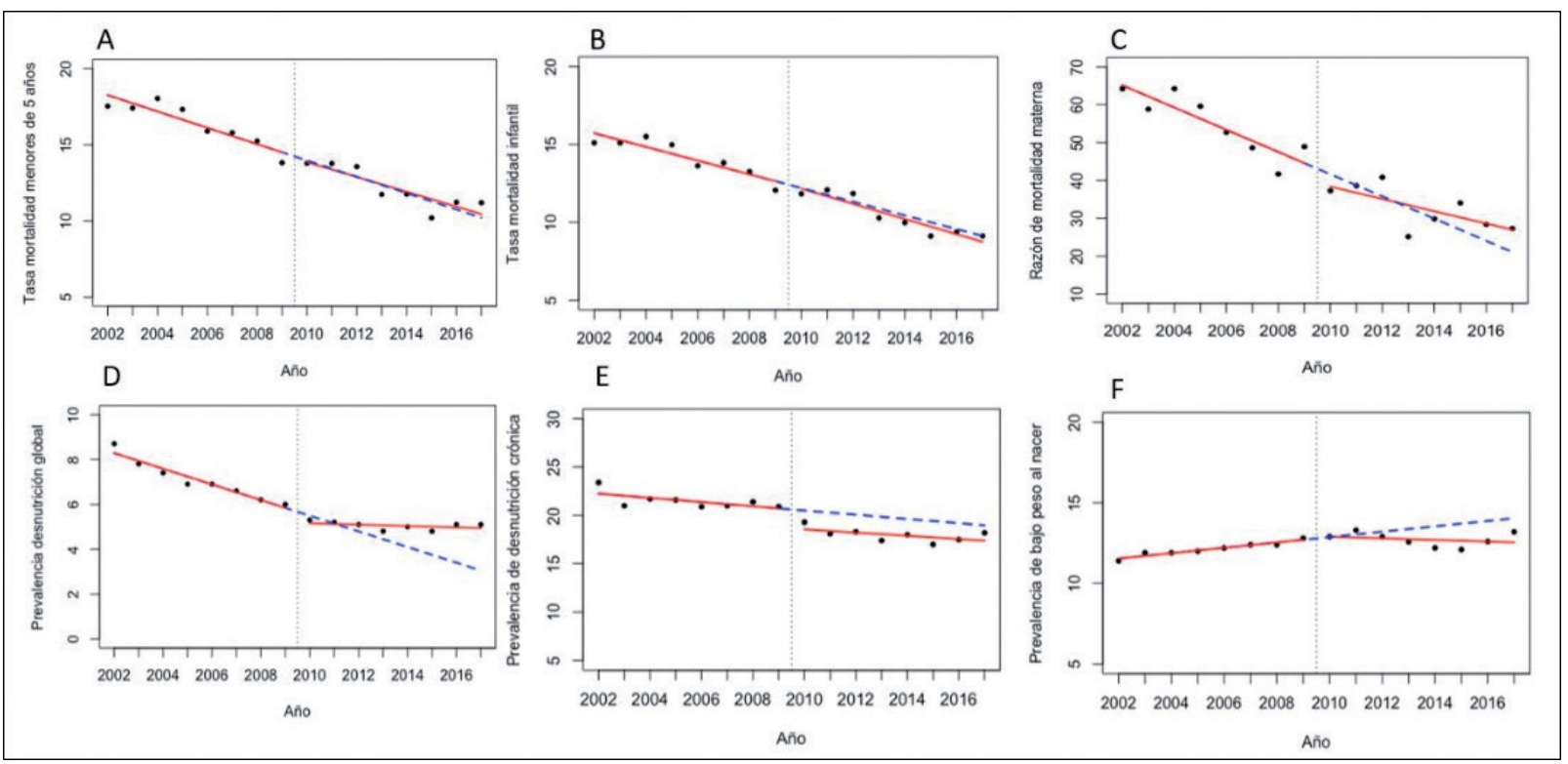

Gráfico 1. Cambio de nivel y tendencia de indicadores de salud antes y después de la implementación de Red Unidos en Bogotá (A) tasa de mortalidad en menores de 5 años por $1.000 \mathrm{NV}$; (B) tasa de mortalidad infantil por $1.000 \mathrm{NV}$; (C) razón de mortalidad materna por $100.000 \mathrm{NV}$; (D) prevalencia de desnutrición global; (E) prevalencia de desnutrición crónica; (F) prevalencia de bajo peso al nacer.

La tasa de mortalidad en menores de 5 años tuvo una reducción significativa por cada año de observación en el período de estudio $(\beta=-0,53 ; \mathrm{p}<0,001)$. Para el inicio del período se ubicó en 17,53 por $1.000 \mathrm{NV}$ $\mathrm{y}$ al final en 11,19 por $1.000 \mathrm{NV}$. No se presentaron cambios estadísticamente significativos de nivel $(\beta=-0,15 ; p=0,82)$ ni de tendencia $(\beta=0,04 ; p=0,75)$ (Ver Gráfico 1-A).

De igual manera, la tasa de mortalidad infantil presentó reducciones significativas por cada año de observación en el período de estudio $(\beta=-0,43 ; p<0,001)$. Al inicio se ubicó en 15,1 por $1000 \mathrm{NV}$, al final llegó a 9,1 por $1.000 \mathrm{NV}$. Al igual que el indicador anterior, no se encontraron cambios significativos de nivel $(\beta=0,006 ; p=0,99)$ ni de tendencia $(\beta=-0,04 ; p=0,68)$. (Ver Gráfico 1-B).

La razón de mortalidad materna presentó reducciones estadísticamente significativas por cada año de observación durante el período de estudio $(\beta=-$ $3,32 ; \mathrm{p}=0,00)$. Para el comienzo del período de observación se ubicó en 64,0 por 100.000 NV y para 2017 en 27,3 por 100.000 NV. Se presentó un cambio de nivel posterior a la intervención $(\beta=-2,61 ; \mathrm{p}<0,05)$, sin embargo, dicha reducción no se sostuvo en el periodo post-intervención $(\beta=1,51 ; p=0,00)$ (Ver Gráfico 1-C).
De igual forma, se presentaron reducciones significativas en la prevalencia de desnutrición global para cada año de observación durante el período de estudio $(\beta=-0,34 ; \mathrm{p}<0,001)$. Al comienzo se ubicó en $8,7 \%$ y para el 2017 se redujo en 3 puntos porcentuales. Posterior a la intervención se presentó un cambio en el comportamiento del indicador $(\beta=-0,65 ; p<0,05)$. No obstante, dicho cambio no se sostuvo en el período post-intervención $(\beta=0,31 ; p<0,001)($ Ver Gráfico 1-D).

En el caso de la prevalencia de desnutrición crónica no se presentaron cambios estadísticamente significativos durante el periodo de observación ( $\beta=-0,21 ; \mathrm{p}=0,052)$. Al comienzo del período se ubicó en $23,4 \%$ y para el 2017 llegó a 18,2\%. En el período post-intervención se presentó un cambio de nivel estadísticamente significativo $(\beta=-2,10 ; \mathrm{p}<0,05)$ pero no de tendencia $(\beta=0,50 ; p=0,72)$ (Ver Gráfico 1-E).

La prevalencia de bajo peso al nacer presentó un aumento estadísticamente significativo por cada año de observación durante el período de estudio $(\beta=0,16 ; p<0,01)$. No presentó cambios de nivel $(\beta=0,23 ; \quad p=0,48)$. Sin embargo, de acuerdo con la tendencia, se presentaron disminuciones estadísticamente significativas en el período postintervención $(\beta=-0,21 ; p<0,05)$ (Ver Gráfico 1-F). 
La estimación de los cambios en nivel y tendencia para los indicadores sociales para Bogotá, antes y después de la implementación de Red Unidos se encuentran en la Tabla 2. La presentación de las series de tiempo interrumpidas se presentan en el Gráfico 2.

Tabla 2. Estimación de cambios de nivel y tendencia de indicadores sociales para Bogotá antes y después de la implementación de Red Unidos.

\begin{tabular}{|c|c|c|c|c|}
\hline Variable & & $\beta$ & IC $95 \%$ & p \\
\hline \multirow{4}{*}{$\begin{array}{l}\text { Pobreza } \\
\text { monetaria }\end{array}$} & Intercepto & 34,85 & & \\
\hline & Tiempo & $-2,1$ & $-2,55 ;-1,64$ & $<0,01$ \\
\hline & Nivel & $-4,42$ & $-7,43 ;-1,41$ & $<0,01$ \\
\hline & Tendencia & 1,70 & 1,$05 ; 2,35$ & $<0,01$ \\
\hline \multirow{4}{*}{$\begin{array}{l}\text { Pobreza } \\
\text { monetaria } \\
\text { extrema }\end{array}$} & Intercepto & 7,50 & & \\
\hline & Tiempo & $-0,58$ & $-0,64 ;-0,52$ & $<0,00$ \\
\hline & Nivel & $-0,64$ & $-1,09 ;-0,20$ & $<0,05$ \\
\hline & Tendencia & 0,55 & 0,$48 ; 0,62$ & $<0,00$ \\
\hline \multirow{4}{*}{$\begin{array}{l}\text { Coeficiente } \\
\text { de Gini }\end{array}$} & Intercepto & 0,57 & & \\
\hline & Tiempo & $-0,005$ & $-0,008 ; 0,002$ & $<0,001$ \\
\hline & Nivel & $-0,004$ & $-0,021 ; 0,012$ & 0,561 \\
\hline & Tendencia & 0,001 & $-0,001 ; 0,005$ & 0,296 \\
\hline \multirow{4}{*}{$\begin{array}{l}\text { Tasa global de } \\
\text { participación } \\
\text { laboral }\end{array}$} & Intercepto & 67,30 & & \\
\hline & Tiempo & $-0,25$ & $-0,67 ; 0,15$ & 0,194 \\
\hline & Nivel & 5,52 & 2,$82 ; 8,21$ & $<0,001$ \\
\hline & Tendencia & 0,31 & $-0,26 ; 0,89$ & 0,255 \\
\hline \multirow{4}{*}{$\begin{array}{l}\text { Tasa de } \\
\text { ocupación }\end{array}$} & Intercepto & 54,95 & & \\
\hline & Tiempo & 0,52 & 0,$08 ; 0,97$ & $<0,05$ \\
\hline & Nivel & 4,64 & 1,$71 ; 7,59$ & $<0,01$ \\
\hline & Tendencia & $-0,43$ & $-1,06 ; 0,19$ & 0,16 \\
\hline \multirow{4}{*}{$\begin{array}{l}\text { Tasa de } \\
\text { desempleo }\end{array}$} & Intercepto & 17,45 & & \\
\hline & Tiempo & $-0,92$ & $-1,12 ;-0,72$ & 0,00 \\
\hline & Nivel & $-1,42$ & $-2,50 ;-0,35$ & $<0,05$ \\
\hline & Tendencia & 1,10 & 0,$79 ; 1,40$ & 0,00 \\
\hline
\end{tabular}

Fuente: elaboración propia a partir del análisis de los datos

La pobreza monetaria presentó una reducción estadísticamente significativa por cada año de observación $(\beta=-2,1 ; \quad p<0,01)$. Para el
2002 se ubicó en 31,7\% y en el 2017 llegó a $12,4 \%$. Se presentó un cambio estadísticamente significativo de nivel $(\beta=-4,42 ; \mathrm{p}<0,01)$. Frente a la tendencia, se evidenció que el cambio que se produjo post-intervención fue hacia un aumento $(\beta=1,70 ; p<0.01)$ (Ver Gráfico 2-A).

La pobreza monetaria extrema presentó una reducción estadísticamente significativa durante el período de estudio $(\beta=-0,58 ; \mathrm{p}<0,00)$. En el 2002 se ubicó en $7,1 \%$ y al final del período en $2,4 \%$. Se presentó cambio de nivel orientada hacia la reducción $(\beta=-0,64 ; p=0,01)$; frente a la tendencia se presentó un aumento estadísticamente significativo en el período post-intervención $(\beta=0,55 ; p<0,00)$ (Ver Gráfico 2-B).

Por su parte, el coeficiente de Gini presentó un cambio estadísticamente significativo para cada año de observación durante el período de estudio $(\beta=-0,005$; $\mathrm{p}<0,001)$. Al comienzo del período fue de 0,571 y para el 2017 fue de 0,498. No se presentaron cambios de nivel ni de tendencia en el período post-implementación (Ver Gráfico 2-C).

No se evidenciaron cambios estadísticamente significativos en la tasa global de participación laboral durante el período de observación $(\beta=-0,25 ; \mathrm{p}=0,194)$. En el 2002 se ubicó en 67,12\% y para el 2017 tuvo un aumento en dos puntos porcentuales. Se presentó un cambio estadísticamente significativo de nivel marcado hacia el aumento $(\beta=5,52 ; p<0,001)$ que no fue sostenido en el período post-intervención ( $\beta=0,31$; $\mathrm{p}=0,25$ ) (Ver Gráfico 2-D).

La tasa de ocupación tuvo un aumento estadísticamente significativo por cada año de observación durante el período de estudio $(\beta=0,52 ; \mathrm{p}<0,05)$. Para el año 2002 se ubicó en 54,88\%, al final del periodo llegó a $62,32 \%$. Se presentó un cambio de nivel posterior a la intervención en términos de aumento $(\beta=4,64 ; p<0,01)$. Sin embargo, no se registraron cambios de tendencia $(\beta=-0,46 ; p=0,16)$ (Ver Gráfico 2-E).

La tasa de desempleo presentó una reducción estadísticamente significativa por cada año de observación durante el período de estudio $(\beta=-0,92$; $\mathrm{p}=0,00$ ). Para el 2002 el $18,24 \%$ de la población bogotana estaba desempleada, para el final del período de observación se ubicó en $10,51 \%$. Se presentó un cambio de nivel $(\beta=-1,42 ; p<0,05)$. En relación con la tendencia, se presentó un aumento posterior a la intervención que fue estadísticamente significativo $(\beta=1,10 ; p=0,00)$ (Ver Gráfico 4-F). 


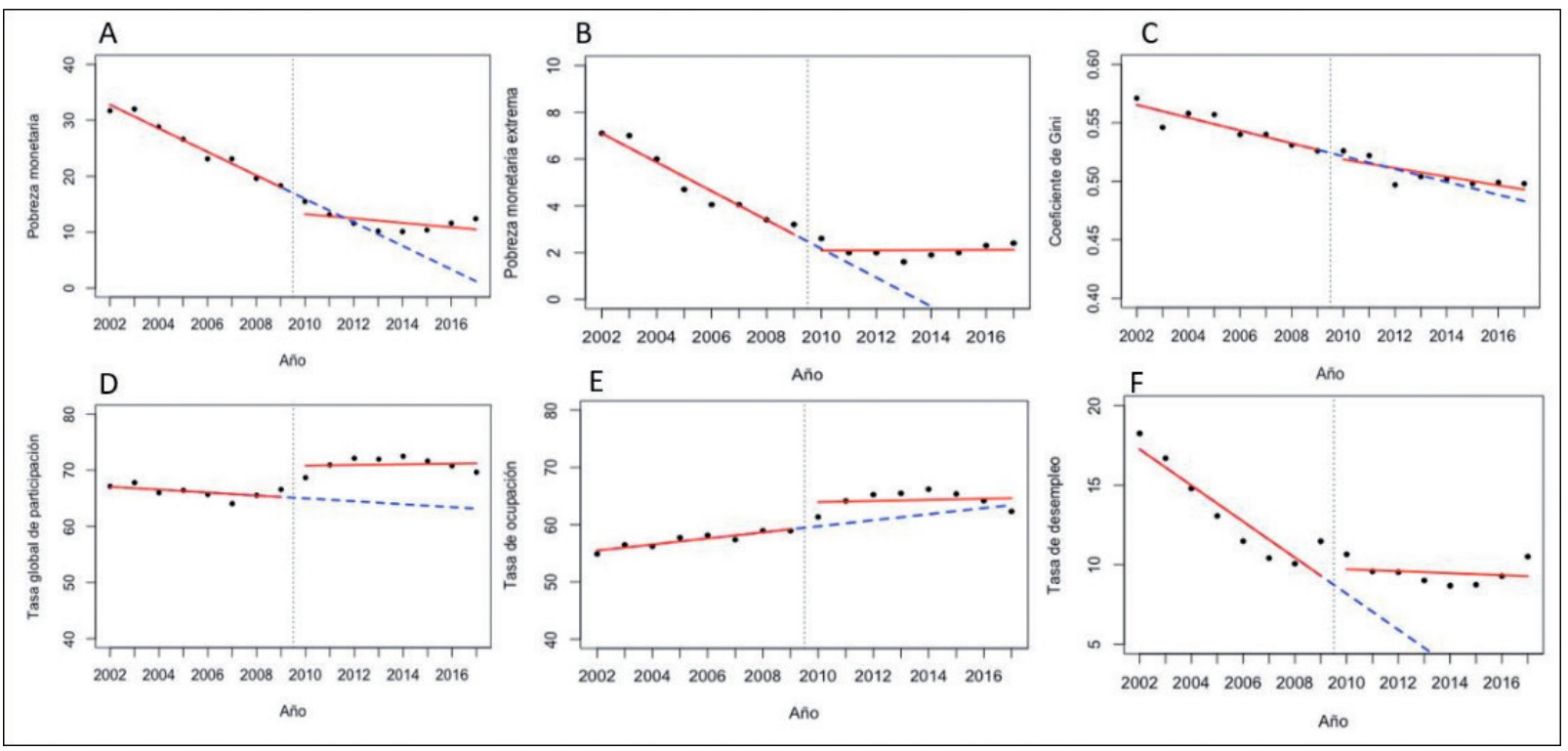

Gráfico 2. Cambio de nivel y tendencia de indicadores sociales antes y después de la implementación de Red Unidos en Bogotá (A) pobreza monetaria; (B) pobreza monetaria extrema; (C) coeficiente de gini; (D) tasa global de participación laboral; (E) tasa de ocupación; (F) tasa de desempleo.

\section{Discusión}

Los indicadores de salud presentaron reducciones significativas por cada año de observación durante el período de estudio, a excepción de la prevalencia de desnutrición crónica. La razón de mortalidad materna y la prevalencia de desnutrición global presentaron cambios significativos posterior a la intervención, sin embargo, dicho cambio no fue persistente en el tiempo. De forma similar, los indicadores sociales presentaron reducciones significativas por cada año de observación durante el período de estudio, a excepción de la tasa global de participación laboral. No obstante, los cambios que se evidenciaron posteriores a la intervención tampoco fueron persistentes en el tiempo.

Las desigualdades sociales y su relación con la salud han sido estudiadas ampliamente ${ }^{33-35}$. Se ha descrito que las normas, las políticas y las prácticas sociales en determinadas situaciones las producen y las reproducen $^{36-38}$. De modo que, la combinación entre políticas y programas sociales deficientes, arreglos económicos injustos y una mala gestión política, son determinantes potenciales para la mala salud ${ }^{36-39}$. Es así como la comisión de los determinantes sociales de la salud ha afirmado que "una buena política pública puede tener efectos beneficiosos en la salud de forma inmediata y a largo plazo"36.
Las políticas orientadas a la reducción de la pobreza podrían mejorar las condiciones de vida de los hogares, lo cual se reflejaría en cambios positivos en las condiciones de vida ${ }^{40,41}$. Sin embargo, los mejoramientos en los indicadores sociales y de salud en este estudio no fueron persistentes en el tiempo.

Se ha encontrado que los programas de asistencia social en países de altos ingresos que tienen como grupo objetivo a las personas en pobreza, no logran resultados en salud positivos en la población beneficiaria. Una razón puede ser metodológica, en la medida que en las investigaciones no se tiene en cuenta el estado de salud de las personas antes de ingresar a este tipo de programas. Otra explicación puede ser que estos programas no proporcionan un mejoramiento real de las condiciones de vida de esta población, sobre todo en términos del ingreso al mercado labora ${ }^{40}$. En esta misma línea, se ha reportado que los impactos de los programas para la pobreza que utilizan Transferencias Condicionadas en Efectivo (TCE) en África Subsahariana, no permiten estimar la persistencia del mejoramiento de las condiciones de salud y sus determinantes en el largo plazo ${ }^{42}$.

Los resultados negativos en la salud infantil están asociados con la pobreza, la falta de protección social, la inseguridad alimentaria y la falta de atención médica. De este modo, los mejoramientos en salud infantil son 
resultado de diversas acciones e intervenciones, entre otras, aquellas orientadas a la reducción de la pobreza. Para el caso de países de medianos y bajos ingresos se ha reportado que los programas para la pobreza tipo TCE son efectivos en el mejoramiento de la salud infantil, sin embargo, para ello se requiere de un efectivo funcionamiento de los sistemas de cuidado en salud ${ }^{41}$. Este mismo hallazgo se reporta en un estudio realizado en Perú, en donde la reducción de la mortalidad materna e infantil, es resultado de una fuerte voluntad política y la implementación de programas intersectoriales contra la pobreza y al mejoramiento de la salud ${ }^{43}$.

El gasto en salud también se ha descrito como una condición que puede mejorar los resultados en salud infantil. Cuando el gasto en salud es público, tiene efectos en la reducción de la mortalidad infantil en países de ingresos bajos, medio-bajos y medio-altos ${ }^{44}$.

Por otra parte, se ha evidenciado una fuerte correlación entre la fuerza económica de un país y menores tasas de mortalidad infantil. Sin embargo, el crecimiento económico no puede ser logrado sin avances en las condiciones socioeconómicas, entre ellas: mejoras en alimentación, en el acceso a la atención en salud y un marco político favorable ${ }^{45}$. De otro modo, se ha descrito que los impactos del crecimiento económico en la pobreza dependen de cuáles son los sectores de la economía que más crecen, el tipo de empleos que se generan y las condiciones iniciales de la economía ${ }^{46}$.

En Bogotá se han venido desarrollando otra serie de programas orientados a reducir las inequidades en los resultados en salud. En 2004 se implementó el programa "salud a su hogar" orientado a mejorar coberturas en afiliación y el mejoramiento de indicadores de salud en hogares de estratos 1 y 2 . Los resultados evidencian reducciones en la mortalidad infantil y en menores de 5 años por nivel de cobertura de la estrategia, entre otros ${ }^{47}$. Por otra parte, existen programas de carácter nacional, como la Red Unidos, y otros de carácter distrital que tienen como objeto de intervención a la población en pobreza. En el caso de Bogotá la secretaria distrital de integración social es la entidad que lidera y formula la política social orientada a reducir las desigualdades económicas, espaciales con primordial atención de aquellas en mayor situación de pobreza y vulnerabilidad $^{48}$.

De este modo, pueden confluir distintos programas y circunstancias más estructurales que afectan a la misma población. Por lo que, de acuerdo con Giner ${ }^{49}$, las acciones sociales, entendidas como políticas públicas, pueden tener resultados esperados o no esperados, en la medida que se implementan en realidades poco conocidas o se adjudican en espacios en donde paralelamente se implementan otras acciones sociales. Es así como no es posible atribuir o establecer relaciones de causalidad entre el comportamiento de los indicadores sociales y de salud con la implementación de la Red Unidos.

Debido a la disponibilidad de los datos, se recurrió a indicadores sociales y de salud para Bogotá y no solamente para aquellos hogares que hicieron parte de Red Unidos. Haciendo la consulta con del Departamento de Prosperidad Social, entidad que lidera la implementación de la Red, sólo se tienen disponible para consulta datos de hogares a partir del 2015.

Puede existir el sesgo de historia ${ }^{30}$, en la medida que otros eventos, diferentes a la implementación de Red Unidos, como los descritos en líneas anteriores, pudieron contribuir a los resultados en los indicadores sociales y de salud en Bogotá durante el período de estudio.

\section{Conclusiones}

Las políticas como la Red Unidos pueden promover el mejoramiento de las condiciones de vida de las personas en extrema pobreza. No obstante, los cambios en los indicadores sociales y de salud en el período posterior a la implementación de la Red Unidos no fueron sostenidos en el tiempo. Sin embargo, estos cambios son producto de múltiples factores que transcurren en otras esferas de la sociedad, no sólo de la Red Unidos.

Desde la salud pública se debe promover que las políticas para la reducción de la pobreza se orienten a configurar sociedades que permitan a sus ciudadanos contar con garantías básicas para el bien vivir. De este modo, cuestiones más estructurales como el mercado de trabajo, el acceso a servicios esenciales para la vida y de formación profesional, de acuerdo con lo que las personas valoren como bueno para sí mismas, serían algunas de las cuestiones que deben abordarse en este tipo de políticas, con el fin de lograr mejoramientos en los indicadores sociales y de salud que se mantengan en el largo plazo.

No obstante, la declaración del éxito o no, de este tipo de medidas, requiere de la disponibilidad de información más precisa. Esto permitiría utilizar otros métodos más específicos de evaluación orientados a evidenciar cambios en las condiciones de vida de las personas que participan de este tipo de intervenciones. 


\section{Consideraciones éticas}

Este artículo hace parte del trabajo de tesis doctoral titulada "Análisis de la política para la superación de la pobreza extrema y el comportamiento de indicadores sociales y de salud a partir de su implementación en Bogotá" aprobada por el Comité de Ética en Humanos de la Universidad CES, registrada en el acta No. 87 de 2015.

\section{Conflicto de interés}

Los autores declararan no tener conflicto de interés

\section{Referencias}

1. Perry G. Prólogo a las actas del taller sobre pobreza y exclusión social en América Latina. En: Exclusión social y reducción de la pobreza en América Latina y el Caribe. Editores: Gacitua E, Sojo C, Davis S. San José de Costa Rica: FLACSO, Banco Mundial; 2000.

2. Pogge TW. World poverty and human rights. $1^{\mathrm{a}}$ ed. United Kingdom: Polity Press; 2008.

3. Katzman R. Seducidos y abandonados: el aislamiento social de los pobres urbano. Rev CEPAL. 2001; 75: 171-189.

4. Retamoso A, Kaztman R. Segregación espacial, empleo y pobreza en Montevideo. Rev CEPAL. 2005; 94: 131-148.

5. Scheil-Adlung X. Response to health inequity: the role of social protection in reducing poverty and achieving equity. Health Promot Int. 2014; 29(Issue suppl 1) i59-i67. doi: https://doi.org/10.1093/ heapro/dau030.

6. Pritchett L, Summers LH. Wealthier is healthier. J Hum Resour. 1996; 31(4) 841-868. doi: https://doi. org/10.2307/146149.

7. Victora CG. Socio-Economic differences in health, nutrition, and population within developing countries: an overview. JAMA. 2007; 298(16): 1943-1949.

8. Sonego M, Pellegrin MC, Becker G, Lazzerini M. Risk factors for mortality from acute lower respiratory infections (ALRI) in children under five years of age in low and middle-income countries: a systematic review and meta-analysis of observational studies. PloS one. 2015; 10(1): e0116380. doi: https://doi.org/10.1371/journal. pone. 0116380 .

9. Pritchard C, Keen S. Child mortality and poverty in three world regions (the West, Asia and Sub-Saharan Africa) 1988-2010: Evidence of relative intra-regional neglect? Scand J Public
Health. 2016; 44(8): 734-741. doi: https://doi. org/10.1177/1403494816675550.

10. Karra M, Subramanian S, Fink G. Height in healthy children in low-and middle-income countries: an assessment. Am J Clin Nutr. 2016; 105(1): 121126. doi: https://doi.org/10.3945/ajcn.116.136705.

11. Banco Mundial. Seguimiento de la cobertura sanitaria universal: informe de monitoreo global 2017. https://apps.who.int/iris/handle/10665/310924.

12. Wagstaff A. Poverty and health sector inequalities. Bull World Health Organ. 2002; 80(2): 97-105.

13. Barrett CB, Garg T, McBride L. Well-being dynamics and poverty traps. An Rev Resource Econo. 2016; 8: 303-27. doi: https://doi.org/10.1146/annurevresource-100815-095235.

14. Banerjee A, Duflo E. Repensar la pobreza. Un giro radical en la lucha contra la desigualdad global. Santillana Ediciones. 2012:1-35.

15. Zibechi R. Contrainsurgencia y miseria. Las políticas de combate a la pobreza en América Latina. Ciudad de México: Pez en el Árbol. 2010.

16. Paura V, Zibecchi C. Los programas de "combate a la pobreza" en las agenda de gobierno y de estudio: condiciones de producción académica, paradigmas y revisiones conceptuales. POSTData: Rev Reflex Análisis Polit. 2018; 23(2): 379-419.

17. Organización de las Naciones Unidas. Objetivos de Desarrollo del Milenio; 2000. https://www.un.org/ es/development/desa/millennium-developmentgoals.html.

18. Organización de las Naciones Unidas. Objetivos de Desarrollo Sostenible; 2015. https://www.undp.org/ content/undp/es/home/sustainable-developmentgoals/goal-1-no-poverty.html.

19. Banco Mundial. La pobreza y la prosperidad compartida 2018: Armando el rompecabezas de la pobreza. Grupo Banco Mundial; 2018. https://openknowledge.worldbank.org/bitstream/ handle/10986/30418/211330ovSP.pdf.

20. República de Colombia. Departamento Administrativo Nacional de Estadística. Pobreza monetaria y multidimensional 2018. DANE; 2019.

21. República de Colombia. Departamento Nacional de Planeación. Conpes social 102. Red de protección social para la superación de la pobreza extrema. DNP; 2006. https://colaboracion.dnp.gov.co/CDT/ Conpes/Social/102.pdf.

22. República de Colombia. Alta consejería para la Prosperidad Social. Plan Nacional para la Prosperidad Social: la hoja de ruta hacia una Colombia sin extrema pobreza 2011. https:// www.minsalud.gov.co/Documentos $\% 20$ y $\% 20$ Publicaciones/PLAN\%20NACIONAL\%20 
DE\%20PROSPERIDAD\%20SOCIAL.pdf

23. República de Colombia. Agencia Nacional para la Superación de la Pobreza Extrema. Resolución 481 de 2014. Por medio de la cual se fijan los criterios de identificación, selección, vinculación, permanencia y egreso de hogares de la Estrategia para la Superación de la Pobreza Extrema- Red Unidos. Diario Oficial No. 49.312 de 22 de 2014.

24. República de Colombia. Departamento Nacional de Planeación. Agencia Nacional para la superación de la pobreza extrema. Evaluación de impacto de Unidos-Red de protección social para la superación de la pobreza extrema. Informe de evaluación para la población en situación de desplazamiento y población focalizada con SISBEN 2011. DNP, ANSPE; 2012.

25. Álvarez-Dardet C. El papel de la epidemiología en la definición de políticas. Hacia la salud pública que se necesita. Informe SESPAS 2008. Gac Sanit. 2008; 22 (Supl 1): 8-13. doi: https://doi.org/10.1016/ S0213-9111(08)76069-2.

26. República de Colombia. Departamento Administrativo Nacional de Estadística. Estadísticas Vitales nacimientos y defunciones. DANE; 2019. https://www.dane.gov.co/index.php/estadisticaspor-tema/demografia-y-poblacion/nacimientos-ydefunciones.

27. República de Colombia. Secretaria Distrital de Planeación. Objetivos de Desarrollo del Milenio. Cierre 2015. Bogotá D.C. SDP; 2016 http://www. sdp.gov.co/system/tdf/repositorio-dice/dice155objdesarrollomilenio-31122016.pdf?file $=1 \&$ type $=$ n ode\&id $=6420 \&$ force $=$.

28. Biglan A, Ary D, Wagenaar AC. The value of interrupted time-series experiments for community intervention research. Prev Sci. 2000; 1(1): 31-49. doi: https://doi.org/10.1023/a:1010024016308.

29. Penfold RB, Zhang F. Use of interrupted time series analysis in evaluating health care quality improvements. Acad Pediatr. 2013; 13(6): S38-S44. doi: https://doi.org/10.1016/j.acap.2013.08.002.

30. Bernal JL, Soumerai S, Gasparrini A. A methodological framework for model selection in interrupted time series studies. J Clin Epidemiol. 2018; 103: 82-91. doi: https://doi.org/10.1016/j. jclinepi.2018.05.026.

31. León Álvarez AL, Betancur Gómez JI, Jaimes Barragán FA, Grisales Romero HdJ. Ronda clínica y epidemiológica: series de tiempo interrumpidas. Iatreia. 2017; 30(3): 344-351. doi: https://doi. org/10.17533/udea.iatreia.v30n3a11.

32. andoc R, Burden AM, Mamdani M, Lévesque LE, Cadarette SM. Interrupted time series analysis in drug utilization research is increasing: systematic review and recommendations. J Clin Epidemiol. 2015; 68(8): 950-956. doi: https://doi.org/10.1016/j. jclinepi.2014.12.018.

33. Orton LC, Pennington A, Nayak S, Sowden A, Petticrew M, White M, et al. What is the evidence that differences in 'control over destiny' lead to socioeconomic inequalities in health? A theory-led systematic review of highquality longitudinal studies on pathways in the living environment. J Epidemiol Community Health. 2019: 73(10): 929-934. doi: https://doi. org/10.1136/jech-2019-212565.

34. Moor I, Spallek J, Richter M. Explaining socioeconomic inequalities in self-rated health: a systematic review of the relative contribution of material, psychosocial and behavioural factors. J Epidemiol Community Health. 2017; 71(6): 565575. doi: https://doi.org/10.1136/jech-2016-207589.

35. Pillas D, Marmot M, Naicker K, Goldblatt P, Morrison J, Pikhart H. Social inequalities in early childhood health and development: a Europeanwide systematic review. Pediatr Res. 2014; 76(5): 418-424. doi: https://doi.org/10.1038/pr.2014.122.

36. Organización Mundial de la Salud. Subsanar las desigualdades en una generación: alcanzar la equidad sanitaria actuando sobre los determinantes sociales de la salud OMS; 2009. https://www. who.int/social_determinants/thecommission/ finalreport/es/.

37. Navarro V. Neoliberalism as a class ideology; or, the political causes of the growth of inequalities. International J Health Services. 2007; 37(1): 47-62.

38. Hernández M. Desigualdad, inequidad e injusticia en el debate actual en salud: posiciones e implicaciones. 2008.

39. Borrell C, Artazcoz L. Las políticas para disminuir las desigualdades en salud. Gac Sanit. 2008; 22(5): 465-473.

40. Warner ET, Gomez SL. Impact of neighborhood racial composition and metropolitan residential segregation on disparities in breast cancer stage at diagnosis and survival between black and white women in California. J Commun Health. 2010; 35(4): 398-408. doi: https://doi.org/10.1007/ s10900-010-9265-2.

41. Owusu-Addo E, Cross R. The impact of conditional cash transfers on child health in low-and middleincome countries: a systematic review. Int J Public Health. 2014; 59(4): 609-618. doi: https://doi. org/10.1007/s00038-014-0570-x.

42. Owusu-Addo E, Renzaho AM, Smith BJ. The impact of cash transfers on social determinants of health and health inequalities in sub-Saharan 
Africa: a systematic review. Health Policy Plann. 2018; 33(5): 675-96. doi: https://doi.org/10.1093/ heapol/czy020.

43. Huicho L, Segura ER, Huayanay-Espinoza CA, de Guzman JN, Restrepo-Méndez MC, Tam Y, et al. Child health and nutrition in Peru within an antipoverty political agenda: a Countdown to 2015 country case study. Lancet Glob Health. 2016; 4(6): e414-e26. doi: https://doi.org/10.1016/S2214109X(16)00085-1.

44. Dhrifi A. Gastos en salud, crecimiento económico y mortalidad infantil: antecedentes de países desarrollados y en desarrollo. Rev CEPAL. 2018; 125: 71-97.

45. Målqvist M. Abolishing inequity, a necessity for poverty reduction and the realisation of child mortality targets. Arch Dis Child. 2015; 100(Suppl 1): S5-S9. doi: http://dx.doi.org/10.1136/ archdischild-2013-305722.

46. Campos Vázquez RM, Monroy-Gómez-Franco LA. La relación entre crecimiento económico y pobreza en México. Investigación económica. 2016; 75(298): 77-113. doi: http://dx.doi.org/10.1016/j. inveco.2016.11.003.

47. Méndez PAM, Hidalgo GG, Romero RV. La estrategia de Atención Primaria en Salud (APS) para Bogotá-Colombia y su relación con la reducción de inequidades de resultados en salud. Rev Gerenc Polít Salud. 2008; 7(14): 88-109.

48. Alcaldía Mayor de Bogotá. Secretaría Distrital de Integración Social. ¿Quiénes somos? http:// www.integracionsocial.gov.co/index.php/entidad/ integracion-social/quienes-somos.

49. Giner S. Sociología. Barcelona: Ediciones Península; 2010. 